\title{
Possible new phase for adjoint QCD
}

\author{
Erich Poppitz \\ Department of Physics, University of Toronto, Toronto, Ontario M5S 1A7, Canada \\ Thomas A. Ryttov ${ }^{\dagger}$ \\ $C P^{3}$-Origins, University of Southern Denmark, Campusvej 55, 5230 Odense M, Denmark
}

(Received 7 May 2019; published 15 November 2019)

\begin{abstract}
We discuss an exotic phase that adjoint QCD possibly exhibits in the deep IR. It is a confining phase, with a light spectrum consisting of massless composite fermions. The discrete chiral symmetry is broken, with unbroken continuous chiral symmetry. We argue that it may give a description of the IR of adjoint QCD with three massless Weyl flavors and that it passes all consistency checks known to us.
\end{abstract}

DOI: 10.1103/PhysRevD.100.091901

\section{INTRODUCTION}

Adjoint QCD has sparked recent interest from a variety of perspectives: applications in beyond the standard model physics, field-theoretic studies of confinement, phenomena emerging in the limit of a large number of colors, and even novel many-body phases [1-9].

Here, we discuss an exotic phase possibly exhibited by adjoint QCD in the deep IR. It is quite simple to describe, yet has not been discussed in the literature. We find it worth filling this gap, especially in light of recent discussions of several conjectured exotic IR phases of two-flavor twocolor adjoint QCD [1-4].

\section{THEORY AND SYMMETRIES}

Adjoint QCD is an $S U\left(n_{c}\right)$ gauge theory with $n_{f}$ Weyl fermions in the adjoint representation of the gauge group. The gauge fields are $A_{\mu}=A_{\mu}^{a} T^{a}$, where $T^{a}$, $a=1, \ldots, n_{c}^{2}-1$, are the generators of the gauge group, while $F_{\mu \nu}=F_{\mu \nu}^{a} T^{a}$ is the field strength tensor. We refer to the gauge fields as gluons and, borrowing language from supersymmetry, refer to the adjoint Weyl fermions $\lambda_{\alpha}^{i}=\lambda_{\alpha}^{a, i} T^{a}, i=1, \ldots n_{f}$, as gluinos $[\mu, \nu, \ldots$ are Lorentz indices, while $\alpha, \beta, \ldots$ are $\operatorname{SL}(2, \mathbb{C})$ spinor indices].

The theory is asymptotically free for $0 \leq n_{f} \leq 5$, where we expect nontrivial IR physics. Starting from the bottom up, the $n_{f}=0$ theory is pure Yang-Mills theory, which confines and generates a mass gap. The $n_{f}=1$ theory is

${ }^{*}$ poppitz@physics.utoronto.ca

ryttov@cp3.sdu.dk

Published by the American Physical Society under the terms of the Creative Commons Attribution 4.0 International license. Further distribution of this work must maintain attribution to the author(s) and the published article's title, journal citation, and DOI. Funded by SCOAP. pure $\mathcal{N}=1$ supersymmetric Yang-Mills theory, which also confines and breaks its discrete chiral symmetry.

On the other hand, if we instead start from the top, both the $n_{f}=5$ and $n_{f}=4$ theories are believed to flow to a nontrivial IR fixed point $[10,11]$. This conclusion is in very good agreement with the majority of lattice simulations, see, for instance, Refs. [6,12-14], as well as recent analytical computations [15-17]. According to standard lore, most features of adjoint QCD are expected to have only little sensitivity to the number of colors through small $n_{c}^{-2}$ corrections [15].

The question remains, however, about what happens to the $n_{f}=3$ theory in the middle of the range, for which much less is known. Only a few lattice simulations exist [18], and it is fair to say that our knowledge of the IR phase of the theory is still very limited. For instance, if one assumes that the theory is at an IR fixed point, then the Padé extrapolated all orders result is $\gamma \sim 1.59$ for the anomalous dimension of the fermion bilinear [17]. This value is large, close to the unitarity bound, and we expect the theory to display an IR phase different from the $n_{f}=4,5$ theories. It is for this $n_{f}=3$ theory that we propose an exotic candidate phase. For now, however, we shall stick to keeping $n_{f}$ arbitrary, although we will eventually settle at $n_{f}=3$. We have little to say about the $n_{f}=2$ theory; a summary of phases recently discussed is in Ref. [4].

Adjoint QCD has a number of global symmetries. Classically, there is a 0-form global symmetry $G^{(0)}=$ $S U_{f}\left(n_{f}\right) \times U(1)$, where the anomalous $U(1)$ is broken to a discrete subgroup, reducing the 0 -form global symmetry $G^{(0)}=S U_{f}\left(n_{f}\right) \times \mathbb{Z}_{2 n_{f} n_{c}}$; identifications of the center of $S U_{f}\left(n_{f}\right)$ with a subgroup of $\mathbb{Z}_{2 n_{f} n_{c}}$ are not shown. The 0 -form symmetry acts on local degrees of freedom (d.o.f.) (fields) in the theory.

Since the fermions are in the adjoint representation, the theory also has a 1-form discrete center symmetry 
$G^{(1)}=\mathbb{Z}_{n_{c}}^{(1)}$. The center symmetry acts on line operators and is related to confinement-if the theory confines fundamental charges, the center symmetry is unbroken.

\section{SUMMARY OF THE PROPOSED IR PHASE}

We suggest that in the deep IR the theory $i$ ) confines, or has an unbroken center symmetry; ii) has massless composite fermions; and iii) has broken discrete chiral symmetry but manifest continuous chiral symmetry. The first point is an assumption, the second is taken care of by finding a set of gauge invariant operators creating composite massless excitations which satisfy 't Hooft anomaly matching of all the 0-form global symmetries, and the last point ensures the 1-form symmetry anomaly matching. Next, we discuss the proposal in more detail.

\section{MASSLESS SPECTRUM AND 0-FORM ANOMALY MATCHING FOR $\boldsymbol{n}_{c}=\mathbf{2}$}

We begin with our proposal for a massless IR spectrum. It is very simple and in a certain sense is a "gauge invariant copy" of the UV fermionic d.o.f. This ensures that all 0 -form 't Hooft anomalies are automatically satisfied.

To be specific, consider $n_{c}=2$ while still keeping $n_{f}$ arbitrary. We propose that the IR is described by the following set of massless composite fermions,

$$
\begin{aligned}
& \left(\mathcal{O}_{1}\right)_{\alpha}^{i}=\operatorname{Tr}\left[F_{\mu \nu}\left(\sigma^{\mu \nu}\right)_{\alpha}^{\beta} \lambda_{\beta}^{i}\right], \\
& \left(\mathcal{O}_{2}\right)_{\alpha}^{i}=\operatorname{Tr}\left[F_{\mu \nu} F_{\rho}^{\nu}\left(\sigma^{\rho \mu}\right)_{\alpha}^{\beta} \lambda_{\beta}^{i}\right], \\
& \left(\mathcal{O}_{3}\right)_{\alpha}^{i}=\operatorname{Tr}\left[F_{\mu \nu} F^{\nu \rho} F_{\rho \sigma}\left(\sigma^{\sigma \mu}\right)_{\alpha}{ }^{\beta} \lambda_{\beta}^{i}\right]_{\alpha}^{i},
\end{aligned}
$$

where the trace is over the adjoint color indices and the Lorentz indices are contracted all the way through in a connected manner.

As can be seen, the proposal essentially consists of the gluinos dressed with an appropriate amount of glue. Since they each contain only a single $\lambda^{i}$, they are all fundamentals under $S U_{f}\left(n_{f}\right)$ flavor and carry charge +1 under discrete chiral $\mathbb{Z}_{4 n_{f}}$ (recall that $n_{c}=2$ ). In Table I, we have summarized the symmetries and charges of both the UV and IR fermions.

Since our proposal for the IR spectrum is a gauge invariant copy of the UV massless fermions, all 't Hooft anomalies of the 0 -form global symmetry $G^{(0)}=$ $S U_{f}\left(n_{f}\right) \times \mathbb{Z}_{4 n_{f}}$ are matched between the UV and IR,

TABLE I. 0 -form symmetries of adjoint QCD for $n_{c}=2$.

\begin{tabular}{lcccc}
\hline \hline & $S U(2)$ & $S U_{f}\left(n_{f}\right)$ & $\mathbb{Z}_{4 n_{f}}$ & $\mathbb{Z}_{2 n_{f}}$ \\
\hline$\lambda$ & Adjoint & $\square$ & 1 & 1 \\
$\mathcal{O}_{1}$ & 1 & $\square$ & 1 & 1 \\
$\mathcal{O}_{2}$ & 1 & $\square$ & 1 & 1 \\
$\mathcal{O}_{3}$ & 1 & $\square$ & 1 & 1 \\
\hline \hline
\end{tabular}

including the relevant mixed anomalies with gravity. For completeness, we enumerate, for $n_{f}>2$, the nonvanishing anomalies matched by the massless fermions $-\lambda$ in the UV and $\mathcal{O}_{1,2,3}$ in the IR- $\left[S U_{f}\left(n_{f}\right)\right]^{3},\left[\mathbb{Z}_{4 n_{f}}\right]^{3}, \mathbb{Z}_{4 n_{f}}\left[S U_{f}\left(n_{f}\right)\right]^{2}$, and $\mathbb{Z}_{4 n_{f}}[G]^{2}$, where $G$ denotes gravity (for $n_{f}=2$, the $\left[S U_{f}\left(n_{f}\right)\right]^{3}$ anomaly should be replaced by the Witten anomaly).

The ability to make a gauge invariant copy of the gluinos is due to their adjoint nature. ${ }^{1}$ The assumed accidental degeneracy and independence of the states created by $\mathcal{O}_{1,2,3}$ are admitted weaknesses of our proposal. Our main defense is its consistency with anomalies, its simplicity, and its elegance. The spectrum also has the advantage that it is easily generalized to arbitrary $n_{c}$. It is possible, however, to construct other solutions, but these are more baroque and less minimal.

Further support for our proposed spectrum may be derived from the fact that the assumed global symmetry realization (unbroken continuous chiral symmetry and broken discrete chiral symmetry) is not a complete fantasy but is actually the one observed in a theoretically controlled study of adjoint QCD. Analytic control over the IR of the theory is achieved upon compactification to $\mathbb{R}^{1,2} \times \mathbb{S}^{1}$, with fermions periodic around the $\mathbb{S}^{1}$, and with the circle size taken small compared to the strong coupling scale of the theory. In the limit of small $\mathbb{S}^{1}$, adjoint QCD can be solved semiclassically $[7,8]$, showing that the symmetries are realized exactly as in our proposal. Thus, our proposal is consistent with a flow to small $\mathbb{S}^{1}$ with no change of symmetry realization. Our conjectured massless spectrum changes upon reducing the $\mathbb{S}^{1}$ size-two of the three massless $S U\left(n_{f}\right)$ fundamentals of (1) obtain chirally symmetric masses at small $\mathbb{S}^{1}$ from their coupling to the center symmetric holonomy.

\section{SYMMETRY REALIZATION AND ANOMALY MATCHING INVOLVING THE 1-FORM SYMMETRY FOR $n_{c}=2$}

It was recently realized that the gauging of 1-form symmetries leads to new 't Hooft anomaly matching conditions $[20,21]$. Here, the relevant anomaly is the mixed $\mathbb{Z}_{4 n_{f}}-\mathbb{Z}_{2}^{(1)}$ discrete chiral/1-form center anomaly.

To detect this anomaly, one introduces a 2 -form center symmetry gauge field background, which changes the topological charge quantization from integer to half-integer [21]. Equivalently, gauging the center symmetry of an $S U(2)$ theory amounts to introducing 't Hooft fluxes, the topological charge of which is half-integer [22,23]. The half-integer topological charge implies that the partition function of the fermions acquires a $\mathbb{Z}_{2}$ phase under a

\footnotetext{
${ }^{1}$ Similar gauge invariants appear in the descriptions of supersymmetric theories with adjoint matter fields, as in Ref. [19].
} 
discrete chiral $\mathbb{Z}_{4 n_{f}}$ transformation. This phase represents the mixed 't Hooft anomaly and has to be matched between the UV and IR of the theory. Our proposed IR spectrum only involves the free fermions created by the local operators $\mathcal{O}_{1,2,3}$. As these local fields are invariant under the 1-form symmetry, anomalies involving center symmetry have to be matched by some other means.

As in Ref. [1], we propose that they are matched in the "Goldstone" mode, with the discrete chiral $\mathbb{Z}_{4 n_{f}}$ suffering a breakdown $\mathbb{Z}_{4 n_{f}} \rightarrow \mathbb{Z}_{2 n_{f}}$, due to the $S U_{f}\left(n_{f}\right)$ invariant expectation value $\left\langle\operatorname{det}_{i j}\left(\epsilon^{\alpha \beta} \lambda_{\beta}^{i a} \lambda_{\alpha}^{j a}\right)\right\rangle$. The anomaly is matched in the IR by an IR topological quantum field theory (TQFT). This is a theory with a finite-dimensional Hilbert space, a "chiral Lagrangian" describing the two degenerate ground states resulting from the breaking of the discrete chiral symmetry. Similar TQFTs arising from the spontaneous breaking of discrete chiral symmetries and matching the mixed anomalies with 1-form symmetries are explicitly described in Refs. [20,24].

Thus, a complete description of the proposed IR phase can be summarized as the theory of the free massless fermions $\mathcal{O}_{1,2,3}$ tensored with the TQFT resulting from the $\mathbb{Z}_{4 n_{f}} \rightarrow \mathbb{Z}_{2 n_{f}}$ chiral symmetry breaking. ${ }^{2}$

\section{GENERALIZING TO ARBITRARY $\boldsymbol{n}_{\boldsymbol{c}}$}

So far, we have discussed the $n_{c}=2$ case, but the generalization of the IR spectrum matching the 0 -form anomalies (1) to arbitrary finite $n_{c}$ is straightforward. We propose that the massless IR spectrum is composed of $n_{c}^{2}-1$ massless fermions made up of gluinos dressed with glue,

$$
\begin{aligned}
\left(\mathcal{O}_{1}\right)_{\alpha}^{i} & =\operatorname{Tr}\left[F_{\mu \nu}\left(\sigma^{\mu \nu}\right)_{\alpha}^{\beta} \lambda_{\beta}^{i}\right] \\
& \vdots \\
\left(\mathcal{O}_{n_{c}^{2}-1}\right)_{\alpha}^{i} & =\operatorname{Tr}[\underbrace{F_{\mu \nu} \cdots F_{\rho \sigma}}_{n_{c}^{2}-1}\left(\sigma^{\sigma \mu}\right)_{\alpha}^{\beta} \lambda_{\beta}^{i}],
\end{aligned}
$$

where again the trace is over the adjoint color indices and the Lorentz indices are contracted all the way through in a connected manner. It is clear that, just as in the $n_{c}=2$ case, these $n_{c}^{2}-1$ gauge invariant operators precisely mirror the UV fermions. They are each a fundamental of $S U_{f}\left(n_{f}\right)$ flavor and carry charge +1 under the discrete chiral $\mathbb{Z}_{2 n_{f} n_{c}}$. Therefore, all 't Hooft anomalies of the 0-form global symmetry are automatically matched between the UV and IR. We propose that these are the massless d.o.f. adjoint QCD exhibits in the deep IR for $n_{f}=3$ and any (finite) $n_{c}$.

\footnotetext{
${ }^{2}$ Note that issues related to the non-Abelian spin-charge relation [2-4] do not arise for $n_{f}=3$, where such a relation is absent.
}

To match the mixed $\mathbb{Z}_{2 n_{f} n_{c}}-\mathbb{Z}_{n_{c}}^{(1)}$ discrete chiral/center anomaly in the IR, we again propose the formation of the $S U_{f}\left(n_{f}\right)$ flavor invariant condensate $\left\langle\operatorname{det}_{i j}\left(\epsilon^{\alpha \beta} \lambda_{\beta}^{i a} \lambda_{\alpha}^{j a}\right)\right\rangle$ breaking $\mathbb{Z}_{2 n_{f} n_{c}} \rightarrow \mathbb{Z}_{2 n_{f}}$. As for $n_{c}=2$, the massless field content of the IR theory should be supplemented with an IR TQFT describing the $n_{c}$ vacua due to the $\mathbb{Z}_{2 n_{f} n_{c}} \rightarrow \mathbb{Z}_{2 n_{f}}$ breaking and matching the $\mathbb{Z}_{2 n_{f} n_{c}}-\mathbb{Z}_{n_{c}}^{(1)}$ anomaly.

Similar to $n_{c}=2$, the main argument in favor of our proposal is consistency of the spectrum and symmetry realization with all anomaly matching conditions known to us. However, the proposed IR spectrum (2), especially when considered for arbitrary large $n_{c}$, has interesting features and raises questions, some of which remain unanswered. We now discuss these, focusing on $n_{f}=3$ :

(1) Nonuniqueness.-We do not expect the solution (2) to be unique. One can construct other massless spectra matching the anomalies using $S U_{f}(3)$ representations different from the $\mathbf{3}$.

(2) Large multiplicities and large anomalous dimensions.-Despite the nonuniqueness, one feature of (2) is likely to be generic to any proposed solution of anomaly matching: the large, $\mathcal{O}\left(n_{c}^{2}\right)$, multiplicity of identical representations, implying that the operators in (2) must acquire large anomalous dimensions to become free in the IR.

To argue this, note that we require $n_{c}^{2}-1$ copies of 3 (of cubic Dynkin index 1) since the $\left[S U_{f}(3)\right]^{3}$ anomaly in the UV theory is $n_{c}^{2}-1$. Whatever other massless IR spectra for Weyl fermions one contemplates, the sum of their cubic Dynkin indices has to add to $n_{c}^{2}-1$. If we take the limit of large (infinite) $n_{c}$, and if we keep the maximum number of boxes in the Young tableau of the $S U_{f}(3)$ representations of the massless fermions finite as we take $n_{c}$ large, their cubic Dynkin indices will be finite, and $\mathcal{O}\left(n_{c}^{2}\right)$ multiplicities will be necessary to match the $\left[S U_{f}(3)\right]^{3}$ anomaly. ${ }^{3}$ Further, in order to have $\mathcal{O}\left(n_{c}^{2}\right)$ multiplicities of an identical representation, the operators that create the different massless states will have to invoke insertions of $F^{k}, k=1, \ldots, \mathcal{O}\left(n_{c}^{2}\right)$ ( $F$ stands for field strength tensors or derivatives), as in (2). While this discussion is not a proof, it suggests that operators of increasingly higher classical dimension [up to $\mathcal{O}\left(n_{c}^{2}\right)$ ] must acquire large anomalous dimensions to become free in the IR.

This may appear unusual, but we recall that similar features have been seen earlier. Consider the duality

\footnotetext{
${ }^{3}$ One can imagine allowing the number of boxes in the Young tableau of the $S U_{f}(3)$ representation to grow with $n_{c}$. We have not studied this in any detail but note that the relevant composite operators will now have up to $n_{c}$ insertions of $\lambda^{i}$, even before taking into account multiplicities needed to match the $\left[S U_{f}(3)\right]^{3}$ anomaly.
} 
of $S U\left(n_{c}\right) \mathcal{N}=1$ supersymmetric QCD with $n_{f}=$ $n_{c}+1$ fundamental flavors [25], the IR phase of which has a similar flavor to our proposed IR phase. First, these are also phases without continuous global chiral symmetry breaking. The massless composite supermultiplets, the mesons $M_{i}^{j}$ and baryons $b^{i}$ and $\tilde{b}_{j}\left(i, j=1, \ldots, n_{c}+1\right)$, saturate 't Hooft anomaly matching at the origin of moduli space. Second, the number of massless composites is $\mathcal{O}\left(n_{c}^{2}\right)$, similar to our proposed phase. The $M_{i}^{j}$ and $b^{i}$ are free in the deep IR, and there is a large emergent global symmetry with rank approximately $n_{c}^{2}$, somewhat similar to our proposal. ${ }^{4}$ Lastly, the composite operators $M, b, \tilde{b}$ develop large anomalous dimensions, as the meson and baryon superfields have classical dimensions 2 and $n_{c}$, respectively, while both become free fields in the IR, as should our operators (2). Of course, the similarity is at most suggestive since we lack the "power of supersymmetry" and the impressive checks of Seiberg's duality.

(3) Large- $n_{c}$ limit.-The operators in (2) —and, as argued above, likely in any other composite-fermion solution of anomaly matching - are of the form $\operatorname{Tr}\left(F^{p} \ldots\right)$, where $p$ can be as large as approximately $n_{c}^{2}$. Usual large- $n_{c}$ counting rules hold for operators where $p$ is kept fixed as $n_{c} \rightarrow \infty$. Notable exceptions are baryon operators in large- $n_{c} \mathrm{QCD}$, understood in Ref. [26], but the physical intuition involved does not apply to our case. Supersymmetric QCD with $n_{f}=n_{c}+1$, as discussed above, is of higher relevance to us. Here the massless baryons $b, \tilde{b}$ involve the product of $n_{c}$ quark superfields. However, we are not aware of a study of the large- $n_{c}$ limit of Seiberg's theory which discusses baryons. ${ }^{5}$

Thus, regarding the fate of our proposal in the large- $n_{c}$ limit, we only note that either the counting is modified to accommodate the operators in (2) (as it probably is for the baryons in supersymmetric QCD) or there is an inconsistency (or perhaps a large- $n_{c}$ phase transition) with our proposed symmetry realization and spectrum in the infinite $n_{c}$ limit. This is a question worthy of future study.

(4) Planar equivalence.- Related to the above discussion, let us juxtapose our proposal with the large- $n_{c}$ planar equivalence between theories A and B $[28,29]$. Theory A is our $S U\left(n_{c}\right)$ adjoint QCD with $n_{f}$ Weyl

\footnotetext{
${ }^{4}$ The emergent global symmetry is broken by IR irrelevant interactions, e.g., those given by the superpotential $W=$ $\operatorname{det} M+b M \tilde{b}$, down to the flavor symmetry group. We also expect this to be the case for the (unknown) interactions between the composite fermions (2).

${ }^{5}$ The study of large- $n_{c}$ counting rules in Seiberg duality [27] considered only the large- $n_{c}$ mesons.
}

flavors. Theory B is an $S U\left(n_{c}\right)$ gauge theory with $n_{f}$ Dirac flavors in the two-index antisymmetric representation. It has been argued, assuming unbroken charge conjugation on $\mathbb{R}^{4}[30]$, that there is a planar equivalence between the bosonic sectors of the theories $A$ and $B$. This already implies that the fermionic operators $\mathcal{O}_{i}, i=1, \ldots, n_{c}^{2}-1$ of theory $A$ cannot be probed using planar equivalence. However, bosonic equivalence alone suggests that the continuous $^{6}$ global symmetry realization of the two theories is the same at $n_{c} \rightarrow \infty$.

One can try to use this equivalence to argue as follows: assume that theory B behaves similarly at $n_{c} \rightarrow \infty$ and $n_{c}=3$. Next, use the fact that theory $\mathrm{B}$ at $n_{c}=3$ is just three-flavor massless QCD with fundamental Dirac fermions, believed to break chiral symmetry [32]. Our assumption then leads us to conclude that at $n_{c} \rightarrow \infty$ the continuous chiral symmetry in theory B should be broken. Then, the planar equivalence would require that theory A also breaks its continuous flavor symmetry in the planar limit, implying that our proposal does not hold at $n_{c} \rightarrow \infty$. While this conclusion might be true, the main assumption, that theory B behaves similarly at $n_{c}=3$ to $n_{c} \rightarrow \infty$, has potential pitfalls, as there may be a phase transition on the way to infinite $n_{c}$. Examples of such behavior can be constructed. For instance, as $n_{c}$ increases, the $n_{f}=6$ theory first enters the conformal window and then loses asymptotic freedom while the $n_{f}=5$ theory enters the conformal window.

\section{SUMMARY OF PROPOSED $\left(n_{f}, n_{c}\right)$ PHASE STRUCTURE OF ADJOINT QCD}

Having now filled the gap for the $n_{f}=3$ theory with a candidate phase, we cannot help but discuss the important characteristics of the emerging phase diagram in the $\left(n_{c}, n_{f}\right)$ plane, ignoring large $n_{c}$ in what follows.

Consider keeping $n_{c}$ fixed, and vary $n_{f}$. For $n_{f}>5$, the theory is IR free, and all 0 -form global symmetries are unbroken. The 1-form center symmetry is broken, due to the perimeter law for the fundamental Wilson loop. As we lower $n_{f}$ to $n_{f}=5$ and $n_{f}=4$, the theory is an interacting conformal field theory in the IR, again with all 0 -form (1-form) global symmetries unbroken (broken). Then, at $n_{f}=3$, massless composite fermions are formed, and discrete chiral symmetry is broken, while continuous chiral symmetry is still unbroken. The theory confines fundamental charges, and the 1-form center symmetry is restored. In addition, there is a TQFT, originating in the discrete symmetry breaking, to match the mixed

\footnotetext{
${ }^{6}$ The matching of discrete symmetry is more subtle; see Ref. [31].
} 
TABLE II. The proposed IR phases of adjoint QCD with $n_{f}$ Weyl flavors.

\begin{tabular}{|c|c|c|c|c|}
\hline$n_{f}$ & IR phase & $\begin{array}{l}\text { Intact } c \chi \\
\text { symmetry }\end{array}$ & $\begin{array}{l}\text { Intact } d \chi \\
\text { symmetry }\end{array}$ & $\begin{array}{c}\text { Intact center } \\
\text { symmetry }\end{array}$ \\
\hline$\geq 6$ & Free & Yes & Yes & No \\
\hline 5 & Fixed point & Yes & Yes & No \\
\hline 4 & Fixed point & Yes & Yes & No \\
\hline 3 & $\begin{array}{l}\text { Confinement, massless } \\
\text { composite fermions }\end{array}$ & Yes & No & Yes \\
\hline 2 & Confinement & No & No & Yes \\
\hline 1 & $N=1 \mathrm{SYM}$ & $\ldots$ & No & Yes \\
\hline 0 & Pure YM & $\ldots$ & $\ldots$ & Yes \\
\hline
\end{tabular}

$\mathbb{Z}_{2 n_{f} n_{c}}-\mathbb{Z}_{n_{c}}^{(1)}$ anomaly. Then, at $n_{f}=2$, the likely [2] (for alternatives, see Ref. [4]) scenario is that a fermion bilinear condensate is formed, breaking both continuous and discrete chiral symmetry. Finally, the $n_{f}=1$ theory is supersymmetric pure Yang-Mills theory with confinement and discrete chiral symmetry breaking.

Loosely speaking, we see that as we lower $n_{f}$ the theory prefers to break more and more of its global 0 -form symmetries. This is somewhat reminiscent of the $\left(n_{f}, n_{c}\right)$ phase diagram of $\mathcal{N}=1$ supersymmetric QCD with fundamental chiral supermultiplets and may be, heuristically, what one expects. A summary of the different IR phases is given in Table II.

\section{ACKNOWLEDGMENTS}

We thank Aleksey Cherman and Mikhail Shifman for discussions and suggestions. E. P. is supported by a Discovery Grant from NSERC. T. A. R. is partially supported by the Danish National Research Foundation under Grant No. DNRF:90.
[1] M. M. Anber and E. Poppitz, Two-flavor adjoint QCD, Phys. Rev. D 98, 034026 (2018).

[2] C. Cordova and T. T. Dumitrescu, Candidate phases for $\mathrm{SU}(2)$ adjoint $\mathrm{QCD}_{4}$ with two flavors from $\mathcal{N}=2$ supersymmetric Yang-Mills theory, arXiv:1806.09592.

[3] Z. Bi and T. Senthil, An adventure in topological phase transitions in 3+1-D: Non-abelian deconfined quantum criticalities and a possible duality, Phys. Rev. X 9, 021034 (2019).

[4] Z. Wan and J. Wang, Adjoint $\mathrm{QCD}_{4}$, deconfined critical phenomena, symmetry-enriched topological quantum field theory, and higher symmetry extension, Phys. Rev. D 99, 065013 (2019).

[5] F. Sannino and K. Tuominen, Orientifold theory dynamics and symmetry breaking, Phys. Rev. D 71, 051901 (2005).

[6] S. Catterall and F. Sannino, Minimal walking on the lattice, Phys. Rev. D 76, 034504 (2007).

[7] M. Unsal, Abelian Duality, Confinement, and Chiral Symmetry Breaking in QCD(adj), Phys. Rev. Lett. 100, 032005 (2008).

[8] M. Unsal, Magnetic bion condensation: A new mechanism of confinement and mass gap in four dimensions, Phys. Rev. D 80, 065001 (2009).

[9] A. Cherman, M. Shifman, and M. Unsal, Bose-Fermi cancellations without supersymmetry, Phys. Rev. D 99, 105001 (2019).

[10] W. E. Caswell, Asymptotic Behavior of Nonabelian Gauge Theories to Two Loop Order, Phys. Rev. Lett. 33, 244 (1974).

[11] T. Banks and A. Zaks, On the phase structure of vector-like gauge theories with massless fermions, Nucl. Phys. B196, 189 (1982).
[12] L. Del Debbio, B. Lucini, A. Patella, C. Pica, and A. Rago, The infrared dynamics of minimal walking technicolor, Phys. Rev. D 82, 014510 (2010).

[13] A. J. Hietanen, K. Rummukainen, and K. Tuominen, Evolution of the coupling constant in SU(2) lattice gauge theory with two adjoint fermions, Phys. Rev. D 80, 094504 (2009).

[14] G. Bergner, P. Giudice, G. Munster, I. Montvay, and S. Piemonte, Spectrum and mass anomalous dimension of SU (2) adjoint QCD with two Dirac flavors, Phys. Rev. D 96, 034504 (2017).

[15] T. A. Ryttov, Consistent Perturbative Fixed Point Calculations in QCD and Supersymmetric QCD, Phys. Rev. Lett. 117, 071601 (2016).

[16] T. A. Ryttov and R. Shrock, Higher-order schemeindependent series expansions of $\gamma_{\bar{\psi} \psi \psi, I R}$ and $\beta_{\mathrm{IR}}^{\prime}$ in conformal field theories, Phys. Rev. D 95, 105004 (2017).

[17] T. A. Ryttov and R. Shrock, Physics of the non-Abelian Coulomb phase: Insights from Pade approximants, Phys. Rev. D 97, 025004 (2018).

[18] G. Bergner, P. Giudice, G. Munster, P. Scior, I. Montvay, and S. Piemonte, Low energy properties of SU(2) gauge theory with $N_{f}=3 / 2$ flavours of adjoint fermions, J. High Energy Phys. 01 (2018) 119.

[19] F. Cachazo, M. R. Douglas, N. Seiberg, and E. Witten, Chiral rings and anomalies in supersymmetric gauge theory, J. High Energy Phys. 12 (2002) 071.

[20] D. Gaiotto, A. Kapustin, N. Seiberg, and B. Willett, Generalized global symmetries, J. High Energy Phys. 02 (2015) 172.

[21] D. Gaiotto, A. Kapustin, Z. Komargodski, and N. Seiberg, Theta, time reversal, and temperature, J. High Energy Phys. 05 (2017) 091. 
[22] G. 't Hooft, A property of electric and magnetic flux in nonabelian gauge theories, Nucl. Phys. B153, 141 (1979).

[23] P. van Baal, Some results for SU(N) gauge fields on the hypertorus, Commun. Math. Phys. 85, 529 (1982).

[24] M. M. Anber and E. Poppitz, Domain walls in high- $T S U(N)$ super Yang-Mills theory and QCD(adj), J. High Energy Phys. 05 (2019) 151.

[25] N. Seiberg, Electric-magnetic duality in supersymmetric nonAbelian gauge theories, Nucl. Phys. B435, 129 (1995).

[26] E. Witten, Baryons in the $1 / n$ Expansion, Nucl. Phys. B160, 57 (1979).

[27] M. Schmaltz, Duality of nonsupersymmetric large $N$ gauge theories, Phys. Rev. D 59, 105018 (1999).
[28] A. Armoni, M. Shifman, and G. Veneziano, Exact results in non-supersymmetric large $\mathrm{N}$ orientifold field theories, Nucl. Phys. B667, 170 (2003).

[29] A. Armoni, M. Shifman, and G. Veneziano, SUSY Relics in One Flavor QCD from a New 1/N Expansion, Phys. Rev. Lett. 91, 191601 (2003).

[30] M. Unsal and L. G. Yaffe, (In)validity of large $N$ orientifold equivalence, Phys. Rev. D 74, 105019 (2006).

[31] A. Armoni, M. Shifman, and M. Unsal, Planar limit of orientifold field theories and emergent center symmetry, Phys. Rev. D 77, 045012 (2008).

[32] J. Preskill and S. Weinberg, 'Decoupling' constraints on massless composite particles, Phys. Rev. D 24, 1059 (1981). 